\title{
Phase Winding a Two-Component BEC in an Elongated Trap: Experimental Observation of Moving Magnetic Orders and Dark-bright Solitons
}

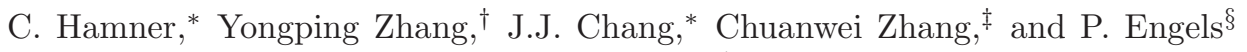 \\ Washington State University, Department of Physics and Astronomy, Pullman, Washington 99164, USA
}

\begin{abstract}
We experimentally investigate the phase winding dynamics of a harmonically trapped twocomponent BEC subject to microwave induced Rabi oscillations between two pseudospin components. While the single-particle dynamics can be explained by mapping the system to a twocomponent Bose-Hubbard model, nonlinearities due to the interatomic repulsion lead to new effects observed in the experiments: In the presence of a linear magnetic field gradient, a qualitatively stable moving magnetic order that is similar to antiferromagnetic order is observed after critical winding is achieved. We also demonstrate how the phase winding can be used to generate copious dark-bright solitons in a two-component BEC, opening the door for new experimental studies of these nonlinear features.
\end{abstract}

PACS numbers: 03.75.Kk, 03.75.Mn, 03.75.Lm, 05.45.Yv

Ferromagnetic and antiferromagnetic $(\mathrm{AF})$ orders are two important and fundamental linear magnetic orders in material physics. For instance, it is well known that AF order exists in the underdoped and low temperature region of the phase diagram for high temperature cuprate superconductors [1]. Ultra-cold atoms provide a clear and highly controllable experimental platform for emulating various condensed matter phenomena. In ultracold atomic gases, AF order has been predicted to exist for both bosons and fermions confined in optical lattices, but reaching the required low temperatures is very difficult [2, 3]. For cold atoms confined in optical lattices, $\mathrm{AF}$ order corresponds to a quantum state where atoms at alternating lattice sites have opposite pseudospins and possess long range phase coherence. For a continuous two-component $\mathrm{BEC}$ in a harmonic trap, there are no such discrete lattice sites, but an AF order can still be defined similarly to that in lattices. Each spin component contains periodic and spatially well separated parts and different spin components appear alternating in space. In lattice system, the lattice periodicity sets the AF length scale, whence for a continuous system the minimum domain spacing is limited by the spin healing length. Twocomponent BECs contain rich physics and have been investigated extensively in the past decade in both experiment and theory [4]. Notable phenomena include the analogy to Josephson junction effects for a BEC in a double well potential [5, 6], the interaction induced phase separation [7, 8], counterflow induced modulational instability [9], novel types of solitons [9], etc.

In this Letter, we experimentally investigate the dynamics of an elongated two-component BEC subject to a Rabi coupling between the two components exposed in the presence of a linear magnetic field gradient. We show that the dynamics can lead to a phase resembling AF order. The strong nonlinear interactions in the BEC play a key role, without them periodic winding/unwinding cycles analogous to the ones of [10,11] are observed instead. We present an insightful mapping to a Bose-Hubbard
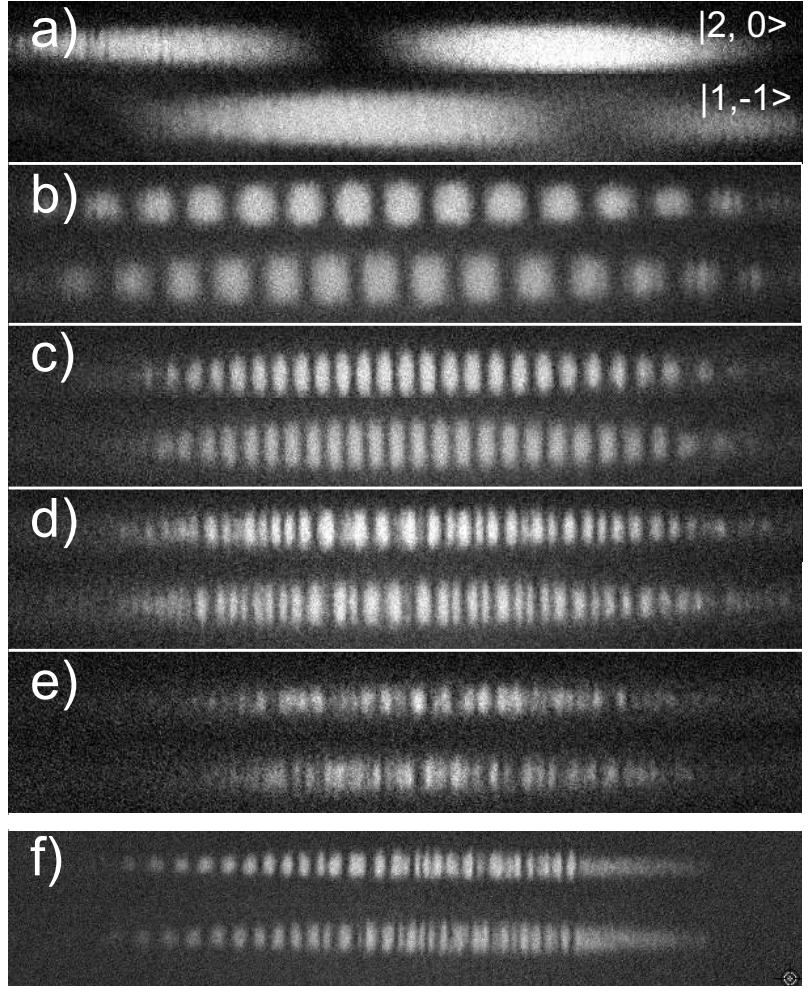

FIG. 1: Phase winding with a detuning gradient across the axial extent of the BEC. The $|2,0\rangle$ state (top cloud in each panel) is coupled to the $|1,-1\rangle$ (bottom cloud) via a microwave pulse of duration a) $10 \mathrm{~ms}$, b) $100 \mathrm{~ms}$, c) $200 \mathrm{~ms}$, d) $300 \mathrm{~ms}$, and e) $900 \mathrm{~ms}$. f) Image taken at larger Raman detuning, but similar gradient, and a winding duration of $600 \mathrm{~ms}$.

model that explains these regular cycles. For the nonlinear case, our experiment and numerics show a succession of stages during the winding. First a period of regular winding exists, which is followed by the emergence of an AF-like pattern, and under the right conditions a dressed state appears. We also demonstrate how such Rabi wind- 
ings can be employed to generate trains of dark-bright solitons.

To showcase the winding dynamics, we start with a BEC containing about $450,000{ }^{87} \mathrm{Rb}$ atoms in the $\left|F, m_{F}\right\rangle$ $=|1,-1\rangle$ hyperfine state. The condensate is confined in an effectively one-dimensional geometry formed by a $1064 \mathrm{~nm}$ single beam optical dipole trap with measured trap frequencies of $\left\{\omega_{x}, \omega_{y}, \omega_{z}\right\}=2 \pi \cdot\{178,145,1.5\} \mathrm{Hz}$. The atoms are coupled to the $|2,0\rangle$ hyperfine state via a microwave pulse of duration $t$. The scattering lengths are $a_{\uparrow \uparrow}=100.4 a_{B}, a_{\downarrow \downarrow}=94.57 a_{B}$, and $a_{\uparrow \downarrow}=98.13 a_{B}$ [12], where $a_{B}$ is the Bohr radius. The choice of the $|1,-1\rangle$ and $|2,0\rangle$ state produces a weakly immiscible system as determined from the mean field condition, $a_{\uparrow \downarrow}^{2}>a_{\uparrow \uparrow} a_{\downarrow \downarrow}$, but similar dynamics are observed for weakly miscible states as well [13]. The strength of the coupling pulse is characterized by the Rabi frequency $\Omega_{0}$ which we measure to be $7.4 \mathrm{kHz}$, when on resonance. The atoms are placed in a magnetic field that consists of a $1 \mathrm{G}$ field in the vertical (y) direction and a magnetic gradient field along the axial $(\mathrm{z})$ direction yielding a z-gradient of about $0.017 \mathrm{G} / \mathrm{cm}$. The gradient provides the means by which the detuning $\delta$, and hence the local Rabi frequency $\Omega$, varies across the cloud [14]. In our experiments, unless noted otherwise the detuning at the center of the BEC is chosen to be $1.3 \mathrm{kHz}$ and the point of zero detuning is located to the left of the BEC in the images. The local Rabi frequency $\Omega_{e f f}=\sqrt{\Omega_{0}^{2}+\Delta^{2}}$ increases as one moves across the BEC to the right. For imaging we use a spin selective technique described in [15]. It involves a short expansion period during which no Rabi drive is applied. Prior to the sudden turn off of the dipole trap for imaging, the two states are vertically overlapped.

While the microwave coupling is applied, windings develop that move in space with a fixed speed set by the local Rabi frequency [16]. The windings move out of the $\mathrm{BEC}$ at one end while new windings emerge at the other end. In Fig. 1(a), a short coupling pulse lasting $t=10 \mathrm{~ms}$ creates only two windings across the cloud. Longer coupling pulses result in more windings with very regular spacing along the axial direction (Fig. 1 (b,c)). Interestingly, for the chosen parameters this increase in the number of windings ceases when the winding duration reaches $t \approx 300 \mathrm{~ms}$, corresponding to an average experimentally observed domain spacing of $15 \mu \mathrm{m}$ and minimum domain spacing of $8 \mu \mathrm{m}$ (Fig. 1(d)). Following this duration the pattern remains qualitatively unchanged for several hundred $\mathrm{ms}$, in the sense that experimental images taken during this interval show domains of similar size, albeit the exact position of the detected domains varies from shot to shot. This long-time behavior is in stark contrast to the behavior observed in less elongated trapping geometries in which the condensate winds and unwinds [10, 11]. After several hundred ms, atom number losses, particularly for the $|2,0\rangle$ state, become significant.

To characterize the formation of the magnetic order,
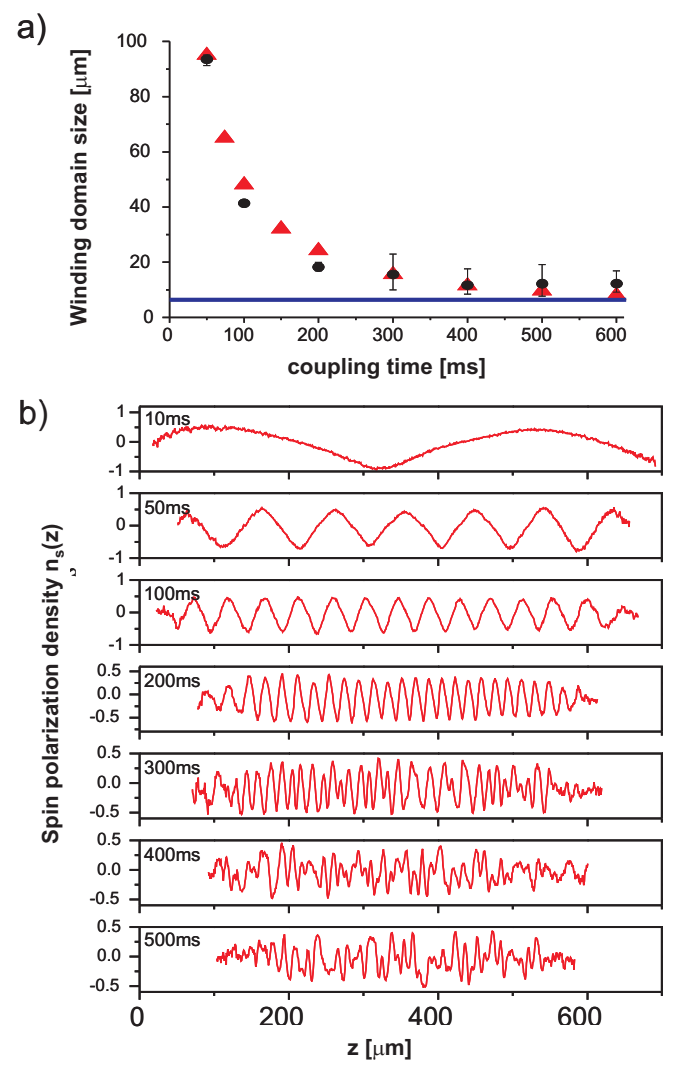

FIG. 2: (a) Saturation of the spatial spin polarization. The domain size, measured in the central region of the BEC, stops decreasing after $\mathrm{t} \approx 300 \mathrm{~ms}$. Overlaid with the experimental results (black circles), where the error bar indicates the variation in the domain size across the central region of the $\mathrm{BEC}$, are numerical results (red triangles). The horizontal line (blue) indicates twice the spin healing length for the initial atom number of the BEC. Panel b) plots the radially integrated spin polarization for the winding durations indicated in each panel.

we plot the minimum domain size of each spin component versus the coupling time in Fig. 2 a). We see that the experimental domain size decreases and then saturates after $t \approx 300 \mathrm{~ms}$. The solid (blue) horizontal line indicates $2 \zeta_{\text {spin }}$ for the initial atom number. For these experimental parameters, the magnetic order becomes qualitatively fixed when the domain size approaches twice the spin healing length $\zeta_{\text {spin }}=1 / \sqrt{8 \pi n|a|}$, where $\mathrm{n}$ is the local density, $a=\left(2 a_{\downarrow \uparrow}-a_{\downarrow \downarrow}-a_{\uparrow \uparrow}\right) / 2$, and $a_{\alpha \beta}$ are the s-wave scattering lengths [17].

In Fig. 2 b), we plot the experimentally observed radially integrated spin polarization density $n_{s}(z)=$ $\left(n_{\uparrow}(z)-n_{\downarrow}(z)\right) /\left(n_{\uparrow}(z)+n_{\downarrow}(z)\right)$ after several different winding durations. The emergence of a magnetic order similar to the AF state, after the Rabi winding saturates, is clearly evidenced by the periodic variation of the spin polarization density in this system. However, $n_{s}(z)$ does not reach unity after a long time (i.e., the 

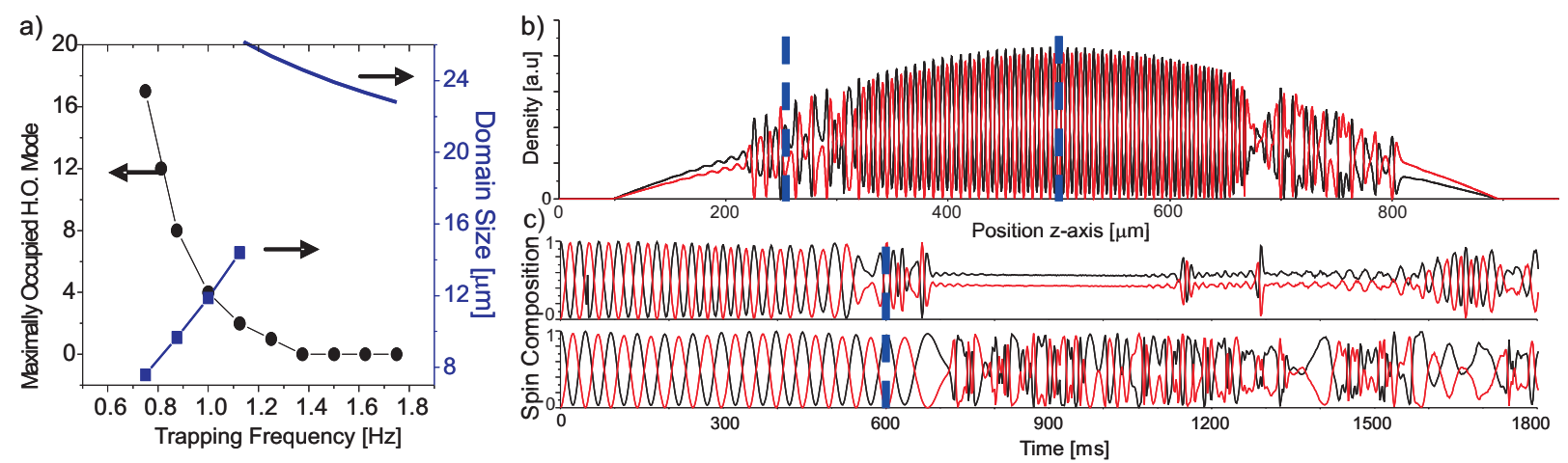

FIG. 3: (a) Effect of the axial confinement on the maximally wound single particle system in the Bose-Hubbard model (black circles). The spin domain size for these maximal windings (blue squares) and twice the harmonic oscillator length (blue solid line) are overlayed. (b,c) Numerical results of the 1D GP equation for experimental parameters. b) Density distribution for the two spin states after $600 \mathrm{~ms}$ of the applied microwave coupling. c) Temporal evolution of the spin populations at $\mathrm{z}=250 \mu \mathrm{m}$ and $\mathrm{z}=500 \mu \mathrm{m}$ for the upper and lower panels respectively. In this graph the chosen $2 \mathrm{~ms}$ timesteps leads to aliasing of the fast and regular Rabi cycles. (color online)

density of one spin component does not fully disappear). We attribute this to the large kinetic energy needed for the complete disappearance of one spin component, to the finite resolution of the imaging system, or to possible expansion dynamics during the TOF imaging. Note that in contrast to AF orders in optical lattices where AF ground states require ultra-low temperature and entropy, the moving AF orders observed here are induced by the external strong Rabi coupling, and the temperature of the BEC plays a negligible role in such dynamics.

For experimental parameters where the spatial variation in the winding rate is increased, a peculiar and qualitatively different behavior can be observed: In this case our experimental as well as numerical studies reveal that extended parts of the BEC can enter a dressed state characterized by the absence of any winding dynamics in both pseudo-spin components [18]. An example is shown in Fig. 1f) where a larger winding rate was produced by choosing a similar magnetic gradient as before but a detuning of $4 \mathrm{kHz}$. After a coupling time of $600 \mathrm{~ms}$ the left edge of the BEC still exhibits Rabi winding while the right edge has evolved into a dressed state. The two regions are separated by a region of qualitatively stable $\mathrm{AF}$ ordering.

The observed dynamics are well reproduced by numerical simulations using the one dimensional GrossPitaevskii (GP) equation. Choosing the units of energy, time and length of the system as $\hbar \omega_{z}, \omega_{z}^{-1}$, and $\sqrt{\hbar / m \omega_{z}}$, we can write the coupled dimensionless equations as

$$
i \frac{\partial}{\partial t}\left(\begin{array}{c}
\Phi_{\uparrow} \\
\Phi_{\downarrow}
\end{array}\right)=\left(\begin{array}{cc}
H_{\uparrow}+\Delta(z) & \Omega_{0} \\
\Omega_{0} & H_{\downarrow}
\end{array}\right)\left(\begin{array}{c}
\Phi_{\uparrow} \\
\Phi_{\downarrow}
\end{array}\right) .
$$

Here, $\Phi_{\uparrow}$ and $\Phi_{\downarrow}$ represent condensate wavefunctions in the hyperfine states $|1,-1\rangle$, and $|2,0\rangle$ respectively. $H_{\uparrow}=H_{0}+2 N \sqrt{m \omega_{x} \omega_{y} / \hbar \omega_{z}}\left(a_{\uparrow \uparrow}\left|\Phi_{\uparrow}\right|^{2}+a_{\uparrow \downarrow}\left|\Phi_{\downarrow}\right|^{2}\right)$, and $H_{\downarrow}=H_{0}+2 N \sqrt{m \omega_{x} \omega_{y} / \hbar \omega_{z}}\left(a_{\downarrow \downarrow}\left|\Phi_{\downarrow}\right|^{2}+a_{\uparrow \downarrow}\left|\Phi_{\uparrow}\right|^{2}\right)$, where
$H_{0}=-\frac{\partial^{2}}{2 \partial z^{2}}+\frac{z^{2}}{2}$, and the second terms describe the mean field nonlinear interaction between atoms. $N$ is total atom number. $\Delta(z)=\Delta_{0}+\delta z$ includes a constant detuning $\Delta_{0}$ and the detuning $\delta$ caused by the magnetic gradient. We numerically solve the GP equation (11) using the experimental parameters as in Fig. 1(a-e) and 2. and determine the domain spacing after various durations of the microwave pulse (red triangles in Fig. 2 a)). We find a good agreement between the numerical results and the experimental data shown. Our numerical simulations also reveal that the spin density polarization $n_{s}(z)$ does not reach unity after a long duration in the $\mathrm{AF}$ phase, agreeing with the experimental observation.

To emphasize the importance of the nonlinear interactions for these dynamics, we contrast the observed behavior with the predictions of the a single-particle picture which ignores meanfield contributions to the spin dynamics. In this case regular winding/unwinding processes exist, which can be understood from an interesting and insightful mapping to a two-component Bose Hubbard model. We start from the linear system of Eq.(1) by neglecting nonlinear terms and expand $\Phi_{\uparrow}$ and $\Phi_{\downarrow}$ using the harmonic oscillator basis $\Psi_{j}, \Phi_{\uparrow}(z)=\sum_{j} a_{j} \Psi_{j}(z)$ and $\Phi_{\downarrow}(z)=\sum_{j} b_{j} \Psi_{j}(z)$. Substituting these expansions into Eq.(1), we obtain

$$
\begin{aligned}
i \frac{\partial a_{j}}{\partial t} & =\frac{1}{2} j a_{j}+\Omega_{0} b_{j}+\Delta_{0} a_{j}+\delta \sqrt{\frac{j+1}{2}} a_{j+1}+\delta \sqrt{\frac{j}{2}} a_{j-1}, \\
i \frac{\partial b_{j}}{\partial t} & =\frac{1}{2} j b_{j}+\Omega_{0} a_{j}
\end{aligned}
$$

which is reminiscent of a two-component Bose-Hubbard model. This model can be interpreted as a lattice system subject to a linear potential $\frac{1}{2} z$, leading to the on-site energy $j / 2$. The linear potential prevents the atom from climbing to large $j$. On the other hand, the effective tunneling coefficient is anisotropic: it is $\delta \sqrt{(j+1) / 2}$ for 
$j \rightarrow j+1$, but $\delta \sqrt{j / 2}$ for $j \rightarrow j-1$. Therefore the atoms prefer to tunnel to large $j$ sites. The competition between the linear potential and the anisotropic tunneling leads to a maximum $j_{0}$. Numerical simulations of Eq.(2) confirm that there exists a maximally occupied $j$ for the dynamical oscillation of $a_{j}$ and $b_{j}$ with the initial condition $a_{0}=1$ and $b_{0}=0$. The $j$-th harmonic oscillator wavefunction has $j$ nodes, yielding $j+1$ possible domains in the density of each component. The maximally occupied mode achieved during the single particle winding is plotted in Fig. 3 a) for various axial confinements. This maximal winding number increases as the axial trap frequency $\omega_{z}$ is decreased while the the magnetic gradient is kept fixed.

The addition of the nonlinearity leads to the coupling of atoms to sites with higher $j$. This is expected based on the following: First, the repulsive interactions lead to a larger spatial extent of the BEC, so that larger modes have to be occupied to reach the same domain spacing. Second, larger interactions reduce $\zeta_{\text {spin }}$, decreasing the minimum possible domain size. The winding dynamics depart from the single particle like recursions when the system attempts to flip its order parameter, to begin unwinding. Fig. 3 (b,c) shows results of the 1D GP simulation for the experimental parameters. Fig. 3b) shows the density profile along the $\mathrm{z}$ axis after $600 \mathrm{~ms}$ winding, i.e. just before the winding fully saturates. Fig. 3 c) shows time evolution plots for the local spin composition at the spatial locations indicated by the vertical lines in $b$ ). The deviations from the single particle like winding occur at different evolution times across the spatial extent of the $\mathrm{BEC}$ and the dynamics across the full extent of the BEC no longer seem synchronized leading to the AF like ordering. We note that in the limit of small nonlinearity the numerics recover the winding/unwinding behavior.

The phase winding can also be exploited as a tool to generate copious dark-bright solitons, enabling studies of their dynamics. During the phase winding, the quantum mechanical phase advances by $\pi$ between consecutive domains of the same component (neglecting a minor deviation from $\pi$ induced by the finite and spatially varying detuning of the Rabi drive). This is a natural starting point for the generation of dark-bright solitons in which a dark soliton, with a phase jump of $\pi$, in one component is filled by a bright soliton in the second component [19]. As we experimentally demonstrate, the phase winding pattern can be transformed into a dark-bright soliton train. For the data shown in Fig. 4 we start by reproducing the situation in Fig.1 (c) and then abruptly turn off the applied gradient as well as the Rabi drive. The subsequent intrap evolution is strongly influenced by the difference in lifetime between the $|1,-1\rangle$ and $|2,0\rangle$ states (30 sec vs 0.5 sec respectively). As atoms are preferentially lost from the $|2,0\rangle$ state, the domains in the $|1,-1\rangle$ state approach each other. Assisted by the phase difference between domains in the $|1,-1\rangle$ state, dark solitons filled by atoms
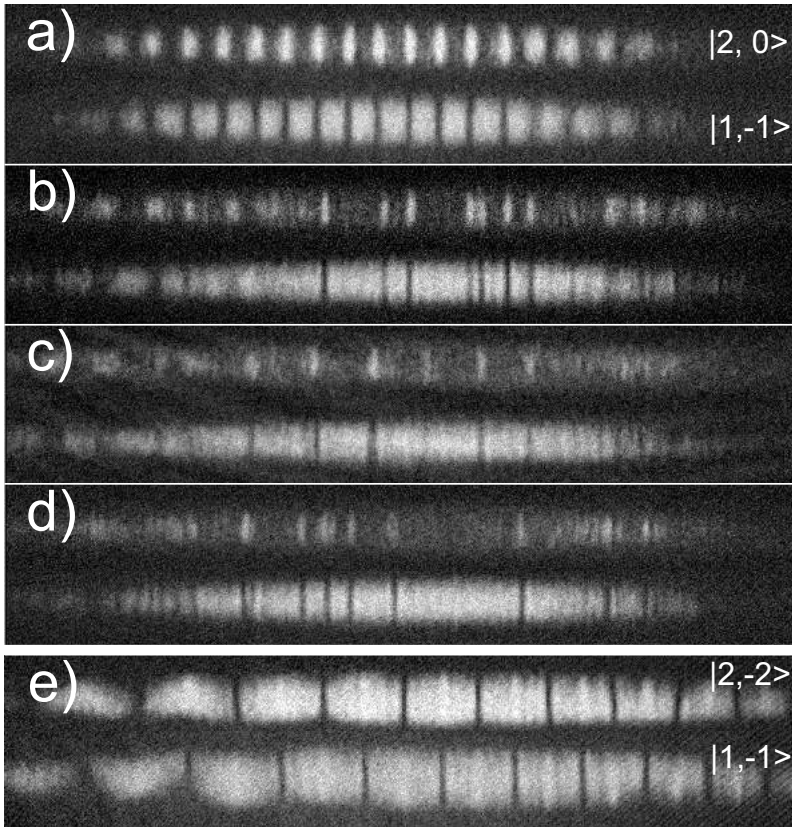

FIG. 4: Generation of dark-bright solitons via phase winding. After applying a coupling pulse of $t=200 \mathrm{~ms}$, the magnetic gradient and coupling are jumped off and the clouds are allowed to evolve in-trap for a) $100 \mathrm{~ms}$, b) $300 \mathrm{~ms}$, c) $400 \mathrm{~ms}$ and d) $500 \mathrm{~ms}$ before imaging. (e) A BEC of atoms in the $|1,-1\rangle$ and $|2,-2\rangle$ state is wound into a soliton train and let evolve for $60 \mathrm{~ms}$. Here a DB soliton train with alternating polarity is generated.

of the $|2,0\rangle$ state form. Figs. 4 (b)-(d) show the evolution of these dark-bright (DB) solitons. The consistent width and long lifetime of these structures provide evidence for their solitonic character as seen under other experimental conditions [15, 20, 21]. Though many DB solitons are formed that are initially equally spaced, the regularity of the arrangement is lost after longer wait times. A similar evolution of the wound BEC into solitons can also be observed for the longer lived states $|1,-1\rangle$ and $|2,-2\rangle$ as seen in Fig. 4 (e) with a 60 ms evolution time after the end of the Rabi drive. Here DB solitons form with alternating polarity in both spin components at the interfaces between the spin domains. To our knowledge, this constitutes the first generation of a train of alternatingly polarized DB solitons in a BEC and offers exciting prospects for the investigation of complex soliton interactions, e.g. the observation of interesting "soliton molecules" [22]. The ability to generate large numbers of dark-bright solitons with well defined initial periodicity may also be an effective starting point for investigations of soliton gases [23, 24].

Acknowledgments: C.H., J.J.C., and P.E. acknowledge financial support from NSF and ARO. Y.Z. and C. Z. acknowledge support from ARO (W911NF-12-1-0334), AFOSR (FA9550-11-1-0313), and NSF-PHY (1249293). 
* These authors contributed equally to this work

$\dagger$ These authors contributed equally to this work; Present address: Quantum Systems Unit, Okinawa Institute of Science and Technology, Okinawa 904-0495, Japan

¥ Electronic address: chuanwei.zhang@utdallas.edu. Present address: Department of Physics, the University of Texas at Dallas, Richardson, TX 75080 USA

$\S$ Electronic address: engels@wsu.edu

[1] P. A. Lee, N. Nagaosa, and X.-G. Wen, Rev. Mod. Phys. 78, 17 (2006).

[2] C. J. M. Mathy, David A. Huse, Randall G. Hulet, Phys. Rev. A 86, 023606(R) (2012).

[3] D. Greif, T. Uehlinger, G. Jotzu, L. Tarruell, T. Esslinger, Science 340, 1307 (2013).

[4] See e.g. M. Abad, A. Recati, arXiv:1301.6864v3; B.J. Dalton, S. Ghanbari, J. Mod. Opt. 59, 287 (2012) and references therein.

[5] J. Williams, R. Walser, J. Cooper, E. Cornell, and M. Holland, Phys. Rev. A 59, R31 (1999).

[6] P. Öhberg and S. Stenholm, Phys. Rev. A 59, 3890 (1999).

[7] D. S. Hall, M. R. Matthews, J. R. Ensher, C. E. Wieman, and E. A. Cornell, Phys. Rev. Lett. 81, 1539 (1998).

[8] K. Sasaki, N. Suzuki, and H. Saito, Phys. Rev. Lett. 83, 033602 (2011).

[9] M. A. Hoefer, J. J. Chang, C. Hamner, and P. Engels, Phys. Rev. A 84, 041605 (2011).

[10] M. R. Matthews, B. P. Anderson, P. C. Haljan, D. S. Hall, M. J. Holland, J. E. Williams, C. E. Wieman, and E. A. Cornell, Phys. Rev. Lett. 83, 3358 (1999).

[11] J. Williams, R. Walser, J. Cooper, E. A. Cornell, and M. Holland, Phys. Rev. A 61, 033612 (2000).

[12] S. J. J. M. F. Kokkelmans, personal communication, (2012).

[13] We have experimentally observed similar winding dy- namics and soliton generation in the longer lived states $|1,-1\rangle$ and $|2,-2\rangle$ which are weakly miscible with scattering length $a_{\downarrow \downarrow}=100.4 a_{0}, a_{\uparrow \uparrow}=98.98 a_{0}$, and $a_{\downarrow \uparrow}=$ $98.98 a_{0}$

[14] In ${ }^{87} \mathrm{Rb}$, the linear Zeeman effect leads to a shift of approximately $700 \mathrm{kHz} /$ Gauss of the $|1,-1\rangle$ state, while the $|2,0\rangle$ state remains unaffected (to first order). The variation of the detuning across the BEC also leads to a variation of the amplitude of the Rabi oscillation, which, however, is negligible for the chosen parameters.

[15] C. Hamner, J. J. Chang, M. A. Hoefer, and P. Engels, Phys. Rev. Lett. 106, 065302 (2011).

[16] A. B. Deb, B. J. Sawyer, and N. Kjrgaard, Phys. Rev. A 88, 063607 (2013).

[17] For our initial atom number the spin healing length at the BEC center is approximately $3.2 \mu \mathrm{m}$.

[18] P. B. Blakie, R.J. Ballagh, C.W. Gardiner, J. Opt. B: Quant. \& Semiclass. Opt. 1, 378 (1999).

[19] B. P. Anderson, P. C. Haljan, C. A. Regal, D. L. Feder, L. A. Collins, C. W. Clark, and E. A. Cornell, Phys. Rev. Lett. 86, 2926 (2001).

[20] C. Becker, S. Stellmer, P. Soltan-Panahi, S. Dörscher, M. Baumert, E. Richter, J. Kronjäger, K. Bongs, and K. Sengstock, Nature Phys. 4, 496 (2008).

[21] S. Middelkamp, J.J. Chang, C. Hamner, R. CarreteroGonzález, P.G. Kevrekidis, V. Achilleos, D.J. Frantzeskakis, P. Schmelcher, P. Engels, Phys. Lett. A. 375, 642 (2011).

[22] D. Yan, J. J. Chang, C. Hamner, P. G. Kevrekidis, P. Engels, V. Achilleos, D. J. Frantzeskakis, R. CarreteroGonzález, and P. Schmelcher, Phys. Rev. A 84, 053630 (2011).

[23] G. A. El, and A. M. Kamchatnov, Phys. Rev. Lett. 95, 204101 (2005).

[24] G. A. El, A. M. Kamchatnov, M. V. Pavlov, S. A. Zykov, J. Nonlin. Sci. 21, 151 (2011). 